\title{
Optimized Selection of Satellite Subsets for a Multi-Constellation GBAS
}

\author{
Daniel Gerbeth, Michael Felux, Mihaela-Simona Circiu and Maria Caamano \\ German Aerospace Center (DLR)
}

\section{BIOGRAPHIES}

Daniel Gerbeth received a Bachelor and Master's degree in Electrical Engineering and Information Technology from Karlsruhe Institute of Technology in 2014. During Master studies he specialized in aerospace technology and navigation. After working in the field of sensor fusion and navigation aiding at Fraunhofer IOSB he joined German Aerospace Center (DLR) in May 2015 and is involved in the research on GBAS now.

Michael Felux received a diploma in Technical Mathematics from the Technische Universität München in 2009. The same year he joined the German Aerospace Center (DLR) where he has was working on the development of the GBAS GAST C testbed at the research airport in Braunschweig and its upgrade to GAST D. He was involved in flight testing and ground validation of the station and the approach procedures. Since 2015 he is coordinating DLRs research on GBAS-based navigation.

Mihaela-Simona Circiu studied Computer Engineering at Technical University Gheorghe Asachi, Romania. She obtained in 2012 a 2nd level Specialized Master in Navigation and Related Application from Politecnico di Torino, Italy. Since 2013 she has been working as a research associate at the Institute of Communications and Navigation at the German Aerospace Center (DLR). Her main focus is multi-frequency multi-constellation Ground Based Augmentation System.

Maria Caamano received a Master's degree in Telecommunications engineering from the University of Oviedo, Spain, in March 2015. The same year she joined the German Aerospace Center (DLR) where she is working on the development of multi-frequency multi-constellation GBAS.

\footnotetext{
ABSTRACT

In the next generation of Ground Based Augmentation System (GBAS) corrections and integrity parameters for two frequencies and multiple constellations will be provided. However, the capacity of the VDB (VHF Data Broadcast) link providing those parameters to users is strongly limited. Thus providing all those new corrections and parameters would either require a longer update rate of corrections or a
}

limitation on the number of satellites to which corrections are provided. In this paper we propose a new satellite selection method which allows fast selection of a variable sized, quasi-optimal subset of all visible GNSS satellites.

The proposed selection heuristic bases on a strong correlation between the third (i.e. vertical) component inside the $S$-matrix for each satellite and the probability of the related satellite being part of a subset which provides a favorable vertical protection level. Utilizing this correlation we design an algorithm which converges fast and leads to optimal results for a majority of constellations. As the selection can be performed for an arbitrary number of satellites and the complexity is not exponentially scaling with the number of available satellites, as an exhaustive search (brute-force) does, the heuristic is flexible and suitable for different applications even beyond GBAS's.

In the context of GBAS these subsets still provide reliable protection levels as the contribution to the accuracy is dropping with every additional satellite. We show the feasibility of using for instance only 14 satellites in global protection level simulations. In a multi-frequency multi-constellation GBAS architecture the approach of selecting only an optimal subset allows to keep the current $2 \mathrm{~Hz}$ update rate. This could mitigate problems with VDB capacity or remove the necessity of additional, more frequent integrity messages.

\section{INTRODUCTION}

The Ground Based Augmentation System (GBAS) provides precision approach guidance for CAT I approaches. In the near future the development and standardization of a CAT II/III capable system will be completed. However, recent studies showed that ionospheric disturbances can potentially have a significant impact on the availability of the service [1]. The introduction of a second usable frequency for aviation on the latest generation of GPS satellites (Block IIF) and the growing Galileo constellation with dual frequency capability provide the possibility to mitigate the ionospheric gradient issue by forming an ionospheric free dual frequency combination of the signals. Furthermore, the larger number of satellites provides improved robustness against ionospheric scintillation or impact of required masking due to suboptimal ground reference station siting. Within the European SESAR project a dual-frequency (DF) dual-constellation (DC) GBAS architecture is currently being developed. 
One of the main constraining factors in the definition of a new DFDC concept is the very limited capacity of the VDB link which is used to provide corrections and integrity parameters to arriving airplanes. Current GPS L1 corrections and integrity parameters still have to be provided in order to allow single frequency GAST-C/D approaches. According to current proposals [2] it is not possible to broadcast all corrections for two frequencies and two (or more) constellations through the current VDB link as backwards compatibility (i.e. for aircraft only equipped with legacy GBAS equipment) has to be ensured. One possibility to overcome this problem is to reduce the update rate for the corrections of the second frequency signals. To fulfill the time to alert requirements, additional integrity messages have to be broadcast with a higher update rate. The integrity concept would have to be revised in order to ensure that with the lower correction update rate all requirements can still be met. In this work we suggest a different solution. While it is beneficial to have more satellites available for navigation, selecting only a subset of the visible satellites could provide the possibility to send corrections for the L5/E5a signals at the standard $2 \mathrm{~Hz}$ update rate.

In order to ensure real-time capability of this approach we present a new satellite selection method. It allows efficient (from a computational point of view) and nearly optimal (regarding the obtained protection levels) selection of a defined number of satellites out of all currently visible. A strong correlation can be observed between the magnitude of the $s_{\text {vert }}$ (the projection factor from the pseudorange into the vertical position) of a satellite inside a constellation and the probability of this satellite being part of an ideal subset. Exploiting this correlation in combination with some prior knowledge from previous sets during continuous operation enables the construction of a straightforward heuristic. This heuristic is analyzed and compared with a computationally very expensive brute-force selection, which always leads to the lowest protection levels by checking all possible subsets at the cost of a significantly higher computation time.

As a measure to compare results the vertical protection level (VPL) is used, as it is the most critical parameter when assessing GBAS performance. Simulations are performed on a global scale, comparing worst case protection levels on a global grid, as well as for selected locations (i.e. airports). Furthermore, subsets fulfilling possible boundary conditions are evaluated e.g. a minimal number of satellites per constellation, or good in-constellation diversity of the geometry. Results show that restricting the corrections to subsets of e.g. 14 optimal selected satellites lead only to minor degradations in protection levels since the information from more satellites often becomes redundant.

Corrections and integrity parameters for these satellite subsets can be provided at the default update rate $(2 \mathrm{~Hz})$ which significantly reduces the necessary effort in terms of integrity validation using a longer update rate. Furthermore, using a heuristic to select the subset instead of brute-force searching for the best set by calculating all resulting protec- tion levels reduces the amount of required computational effort by up to five orders of magnitude.

We start with a short discussion on protection level calculation in GBAS in Section 2. After that some basics on satellite selection are given in Section 3 and the developed heuristic itself is presented. In Section 4 the impact on protection levels from a geometrical point of view when using only a limited number of satellites as compared to two full constellations is examined. Section 5 finally concludes with the protection levels achieved using the new heuristic and performance improvement as well as a short outlook to further studies.

\section{GBAS PROTECTION LEVEL BACKGROUND}

\section{VPL Calculation}

First, a short overview shall be given about the computation of protection levels which are later on used as measure to assess the new satellite selection method. These protection levels define a bound on the GBAS position error with the required probability of $1-2 \cdot 10^{-7}$ according to [3]. The system availability during an approach is then measured by comparing the protection levels in horizontal and vertical direction with the according, distance-related alert limits. These represent the largest value a protection level may take for which the operation is still considered safe. As vertical protection is both harder to fulfill and more critical during the landing, the VPL will be utilized in the further studies. Following RTCA DO-253C [4] the VPL in the fault-free (i.e. no receiver faults) case is calculated as:

$$
V P L_{A p r_{-} H 0}=k_{f f m d} \cdot \sqrt{\sum_{i=1}^{N} s_{v e r t, i}^{2} \cdot \sigma_{i}^{2}}+D_{V}
$$

where $\sigma^{2}$ consists of different error contributions

$$
\sigma_{i}^{2}=\sigma_{p r_{-} g n d_{-} x, i}^{2}+\sigma_{\text {tropo }, i}^{2}+\sigma_{p r_{-} a i r, i}^{2}+\sigma_{\text {iono }, i}^{2}
$$

and

$$
s_{\text {vert }, i}=s_{3, i}+\tan \left(\theta_{G P A}\right) \cdot s_{1, i} .
$$

Index $i$ indicates the $\mathrm{i}$-th satellites of the $N$ used for VPL computation. More detailed information on the different error contributions can be found in [4]. The fault-free missed detection multiplier $k_{f f m d}$ is set to 5.81 according to [4], fulfilling the above mentioned integrity constraints for a base station with three reference receivers. $D_{V}$, the vertical difference in a position solution based on $100 \mathrm{~s}$ and another position solution based on $30 \mathrm{~s}$ smoothed pseudoranges, is not considered in this study as it depends on the approach type and the ionospheric conditions during the approach. As glide path angle $\left(\theta_{G P A}\right) 3^{\circ}$ are assumed. Furthermore, all VPL calculations in this paper assume no faults in the receivers $\left(\mathrm{H}_{0}\right.$ hypothesis), VPLs for the faulted case are not considered. 

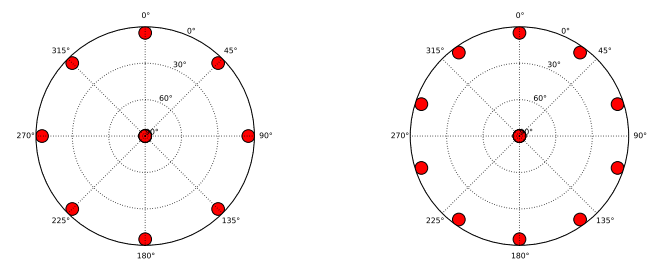

Figure 1: Optimal geometry for 12 and 14 satellites. Both times four satellites are in the center.

\section{Simulation Setup}

The following additional assumptions are underlying all the performed VPL simulations unless otherwise stated:

- $5 \mathrm{~km}$ distance to the GBAS reference point

- $15 \mathrm{~m}(\simeq 50 \mathrm{ft})$ height difference to the GBAS reference point

- $5^{\circ}$ elevation masking

- aircraft velocity of $70 \mathrm{~m} / \mathrm{s}$

- $\sigma_{g n d}$ values calculated from measurements of DLR GBAS testbed in Braunschweig, Germany ([5][6])

- no significant ionospheric disturbances present

- current GPS (31 satellites) and nominal future Galileo (27 satellites) constellation

Based on VPLs computed in the above mentioned way and with the given assumptions all following comparisons and evaluations will be carried out. When talking about best possible subsets in the subsequent sections, the achieved VPL is always taken as measure to define optimality.

Another general aspect shall be mentioned in advance. Most of the comparisons in this paper are based on protection levels averaged over time. This is due to the fact that, although in reality the worst cases have to be considered, these worst case protection levels are highly depending on e.g. small fluctuations in the orbits. Therefore, simulated maximum protection levels (e.g. within one week) would be significantly less meaningful considering the main focus of this research.

\section{SATELLITE SELECTION HEURISTIC}

\section{Background}

In this section a brief background on general satellite selection methods shall be given now. As previous studies have shown ([7], [8]) the best possible satellite geometry in terms of achieved DOP (Dilution of Precision) is defined by a number of satellites with the highest possible elevation and all other satellites equally spaced (in azimuth direction) as close to the horizon as possible (Figure 1). This is problematic in that regard that satellites very close to the horizon commonly experience higher multipath as well as more ionospheric and tropospheric disturbances [9]. These deteriorations of the signals from low elevation satellites are not taken into account by most established algorithms.
They commonly only work with the geometry itself ([7], [8], [10]-[13]) and therefore assume all ranging signals to be equally precise, which is usually not the case. Furthermore, the way many methods work makes it hard to introduce any kind of weighting in the selection process. Some other existing selection algorithms suffer from the additional constraint of a fixed set size ([14]-[16]), which makes them unapplicable for GBAS.

All these issues are solved, when basing the selection algorithm on the pseudoinverse $\mathbf{S}$ of the weighted geometry matrix $\mathbf{G}$ containing all available satellites:

$$
\mathbf{S}=\left(\mathbf{G}^{T} \mathbf{W G}\right)^{-1} \mathbf{G}^{T} \mathbf{W}
$$

where each row $\mathbf{G}_{i}$ of $\mathbf{G}$ is defined as

$$
\left[-\cos \left(E l_{i}\right) \cos \left(A z_{i}\right)-\cos \left(E l_{i}\right) \sin \left(A z_{i}\right)-\sin \left(E l_{i}\right)\right. \text { 1] }
$$

with $A z_{i}$ and $E l_{i}$ being the azimuth and elevation of the i-th satellite respectively.

As a weighting can be defined for every single satellite, through the diagonal elements of matrix $\mathbf{W}$, the satellite selection can be adjusted to different requirements. This weighting can be for example elevation based, $\sigma$-based as commonly in GBAS or using actual measurements.

In the context of GBAS one could argue that ground station and arriving aircraft not necessarily see the same satellites. The ground station could select satellites which an arriving aircraft is not tracking. Although this is theoretically the case, even $40 \mathrm{~km}$ from the ground station the largest possible difference in elevation is approximately 0.35 degrees. The additional elevation difference resulting from the different height of ground station and aircraft is negligible in the range of $10^{-3}$ degrees during final approach. Therefore it is unlikely that many selected satellites are only visible to the ground station but not to aircraft around.

If necessary, this problem can easily be solved by using a slightly increased nominal elevation mask in the ground station compared to the airborne system. This could additionally make sure that arriving aircraft are tracking all satellites (for which corrections are provided) long enough for all smoothing filters to be converged [17]. Unfortunately another, more relevant, reason for an aircraft to lose track of satellites during approach can not be addressed this way. When maneuvering, especially banking, low elevation satellites might be shadowed by parts of the airframe. This renders them unusable for at least the convergence time which can affect availability during approach. Whether this effect would become more critical when only a limited number of satellites is used could be investigated in further studies.

\section{Algorithm}

In the following part the algorithm enabling efficient selection of satellites which provide a small VPL is described. The proposed method itself is mainly based on the very high correlation between the magnitude of the $s_{\text {vert }}$ (Equation (3)) of a satellite inside a (full) constellation and the 


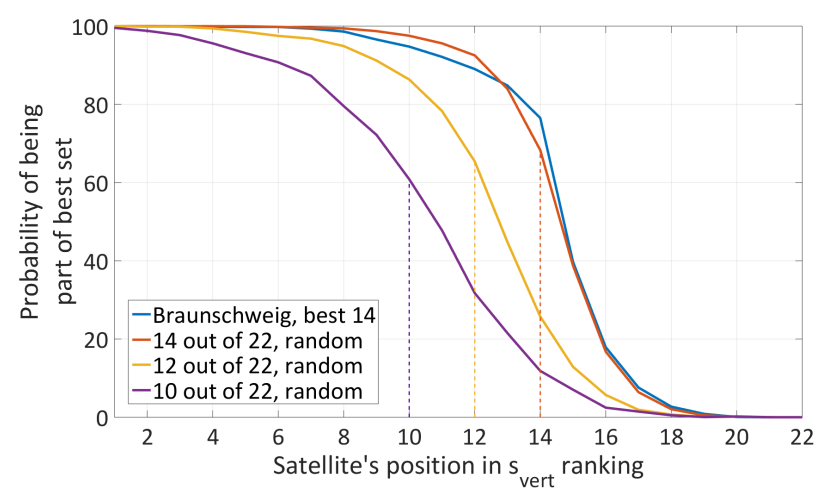

Figure 2: Probability of a satellite being part of an optimal subset depending on the position in the $s_{\text {vert }}$ ranking.

probability of this satellite being part of an optimal subset. In other words: ranking all satellites by descending $\left|s_{\text {vert }}\right|$ and taking the first $n$ elements of this ranking already leads to a very good subset of $n$ satellites in most cases.

This can be seen in Figure 2 which shows the probability of a satellite in a sorted $s_{v e r t}$-ranking to be in a best possible subset for different set sizes. Taking for example a satellite at rank 8 , the probability is around $80 \%$ for being part of a best-10 set and almost $95 \%$ for a best- 12 set. In the case of Braunschweig 14 satellites were selected out of the visible GPS and Galileo satellites over one day (8640 samples). For the three remaining graphs 5000 constellations each with 22 randomly placed visible satellites were generated and 10, 12 and 14 satellites selected. No real orbits were considered in this case, the satellite were uniformly distributed in azimuth and normally distributed in elevation to get random GNSSlike constellations. Ground truths were always generated by brute-force checking all possible subsets and selecting the best one. The steep drop of the probability around the size of the subsets gives a good indication that a reasonable starting set can be found through the aforementioned approach.

Additionally two more starting sets are considered in the algorithm: one is the mere geometry, not taking into account the weighting matrix, which in some situations (5-10\% of the cases) leads to a better VPL. The second alternative, if applicable e.g. in continuous processing, is the best set from the previous time. This is obvious, as the geometry evolves slowly over time and a constellation from seconds ago is (if all satellites are still visible) therefore quite likely again a good choice. And even if for example one satellite, which was part of the subset last time, is no longer available, it is still reasonable to use the remaining satellites. The gap is in this case filled with the necessary number of remaining satellites. One option would be to simply use the remaining satellites by their position in the ranking.

The best of these three subsets is then used as a starting point for further optimization. An overview of the complete process is illustrated in Figure 3. The optimization is again utilizing the probability distribution from Figure 2. Instead of trying to exchange satellites randomly, it is most promising to try exchanges on the last ones in the $s_{\text {vert }}$-ranking first, as these have the highest chance to be not part of the best set. Furthermore, the best approach is to exchange a

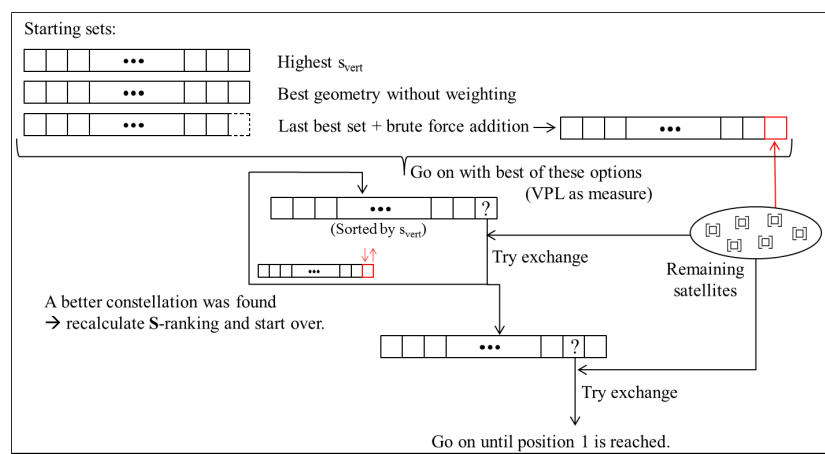

Figure 3: Overview of the selection algorithm.

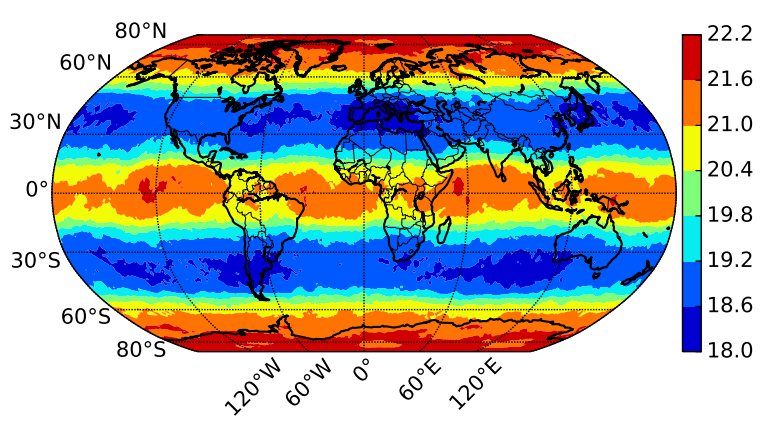

Figure 4: Average number of visible satellites using GPS and Galileo combined (elevation $>5^{\circ}$ ).

satellite with the remaining satellites with the largest $\left|s_{\text {vert }}\right|$ (in the full constellation) first, to shorten the convergence time even more.

The optimization cycle proceeds as follows:

1. Calculating the $\mathbf{S}$ matrix for the current subset.

2. Generating the descending $s_{\text {vert }}$-ranking.

3. Starting from the last position in the ranking each satellite is exchanged with all the remaining ones not in the subset.

4. If a subset is found which provides a better VPL the process starts over from step (1).

5. When the optimization reaches the first satellite and no better subset was found anymore, the process is finished.

This procedure makes sure, that there is no more improvement possible by the exchange of a single satellite. The Hamming distance to a better set (if there exists one) is at least 2. Now the same algorithm could be utilized to go further and check also the sets with a distance of two, but this already increases the computation complexity enormously. Moreover, as results will show later, the possible gain (at least in the context of GBAS) is virtually negligible.

\section{PROTECTION LEVEL COMPARISONS}

First, we will take a look at the average number of satellites visible using GPS and Galileo combined. As can be seen from Figure 4 the global mean averages around 19 


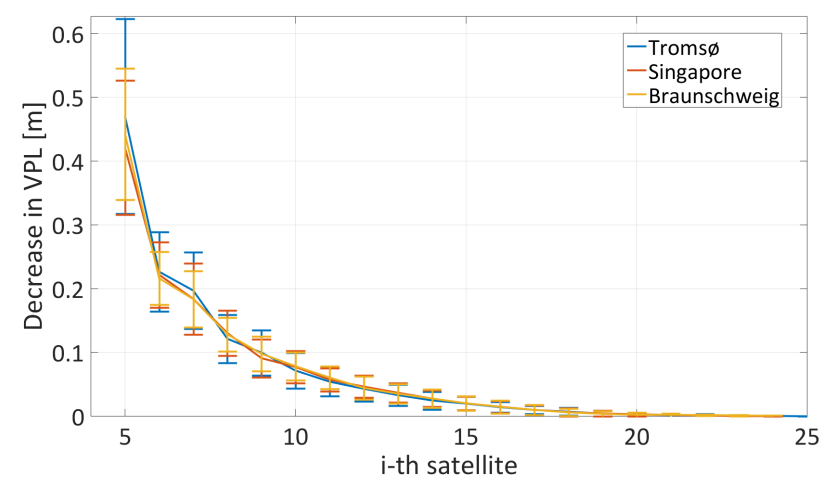

Figure 5: Decrease in vertical protection level per additional satellite for different locations.

satellites in medium latitudes, ranging from 18 to 22 on a global scale. While constraining the number of used satellites from that down to e.g. 14 seems major in the first place (it shrinks the number by up to $36 \%$ ) this becomes much less critical, when considering Figure 5. Due to the exponentially dropping contribution of every additional satellite to the protection level, the actual loss is much smaller than intuitively expected. For the three plotted locations in different latitudes the behavior is basically identical and even independent of the total number of available satellites. On average the contribution from the satellites above 14 in the ranking accounts for less than $5 \%$ of the total gained VPL from satellite 5 on. In other words: selecting a good subset of 14 satellites out of any number available on average still leaves you with $95 \%$ of the initial accuracy.

This can likewise be seen in the global VPL simulations. Figure 6 shows the average achieved protection levels during a 10-days simulation using a GPS/Galileo dual constellation. Based on this, Figures 7 and 8 show the increase in VPL when using only 12 or 14 satellites. The subsets were selected to achieve the best VPLs utilizing brute-force search. Using only the best 12 satellites leads to a degradation of 8 to $23 \mathrm{~cm}$ while the values stay below $15 \mathrm{~cm}$ for $95 \%$ of the globe. Compared to the $10 \mathrm{~m}$ vertical alert limit (VAL), which is not allowed to be exceeded during final GBAS approaches [3], the effect is already quite small. Consequently this is even more distinct, when raising the number of used satellites to 14 . Here the average increase ends up between 1.5 and $12 \mathrm{~cm}$, staying below $5 \mathrm{~cm}$ for medium latitudes. A significant influence on the availability is therefore very unlikely to occur from a limitation to this number of satellites.

Finally, Figure 11 shows an overview of the global achieved average protection levels using different set sizes. The graphs show the VPL for the given set sizes by averaging over all longitudes. This gives a good indication of the (dominating) latitude dependency and again shows the merely minor improvements from 14 satellites onwards. In conclusion the results indicate, that using only 14 (or even 12) satellites in a dual-frequency, dual-constellation context of GBAS would still provide sufficient VPLs.

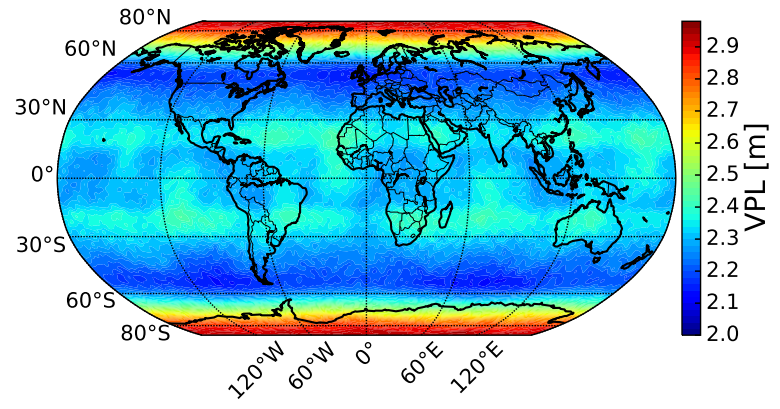

Figure 6: Global VPL (average over 10 days) using all available satellites in view.

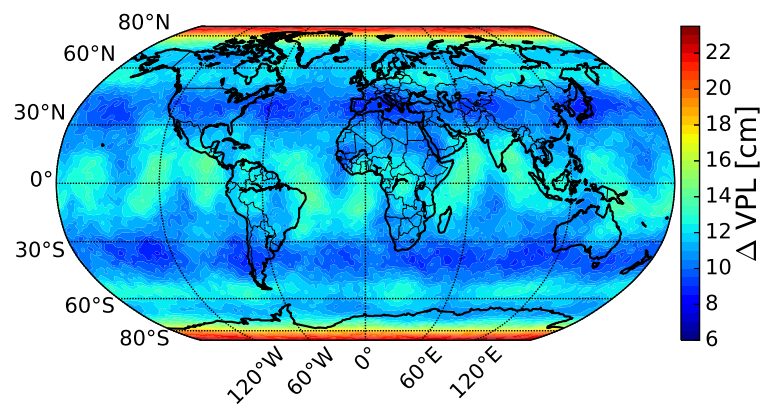

Figure 7: Increase in VPL comparing 12 used satellites to all in view (Fig. 6).

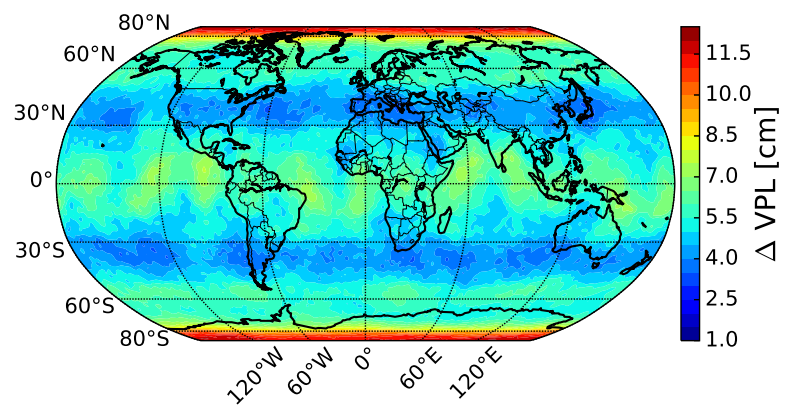

Figure 8: Increase in VPL comparing 14 used satellites to all in view (Fig. 6). 


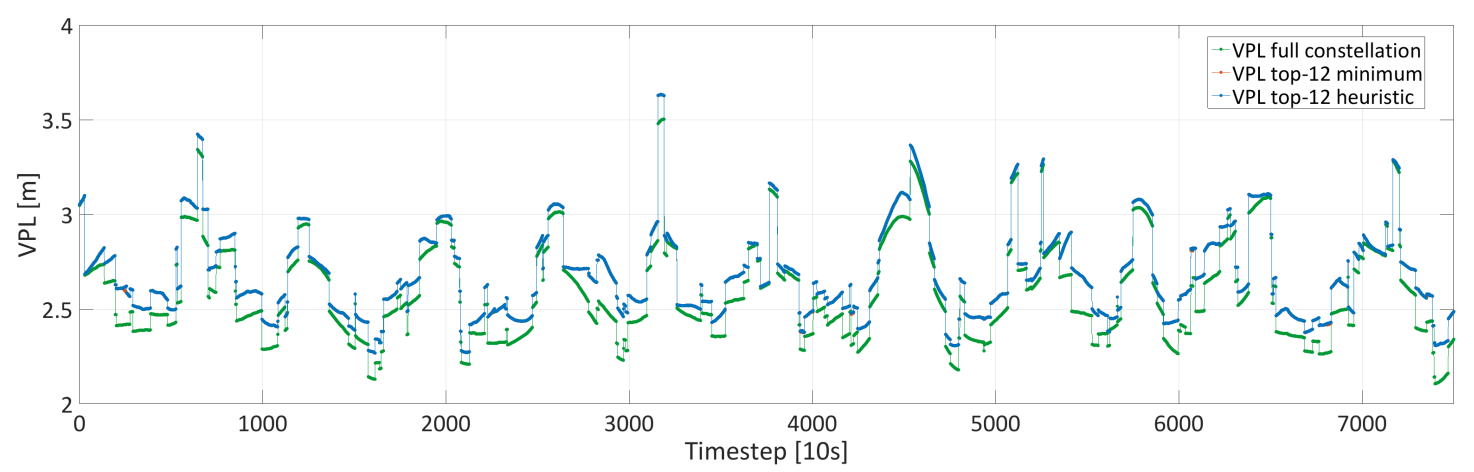

Figure 9: Comparison of achieved VPL using proposed heuristic, brute-force selected best set and all satellites in view.

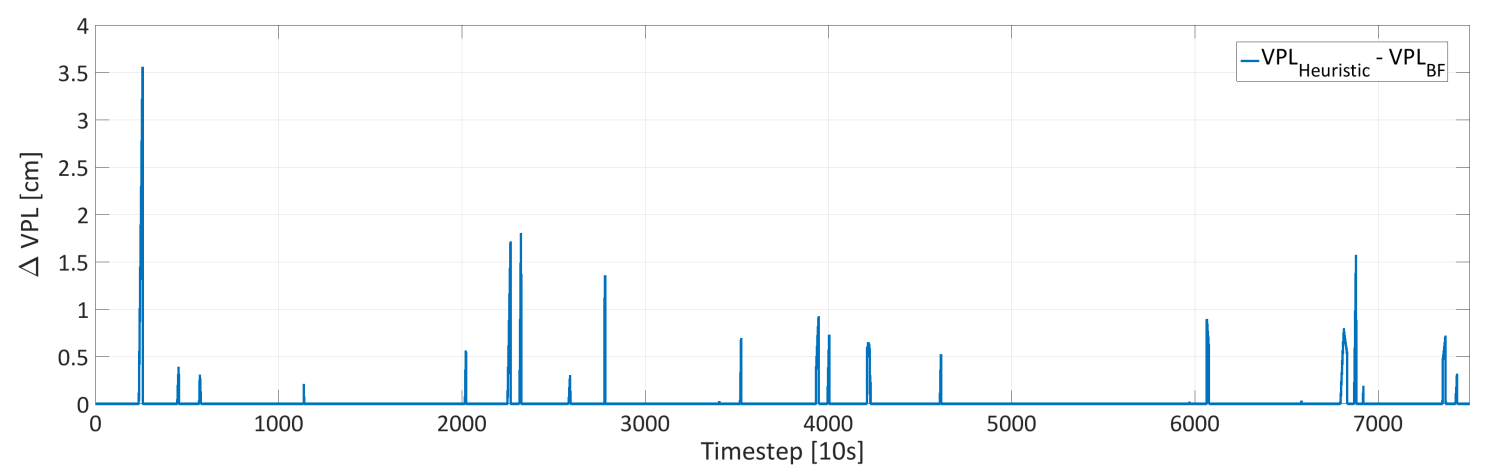

Figure 10: Difference in achieved VPL for brute-force selected best set and proposed heuristic.

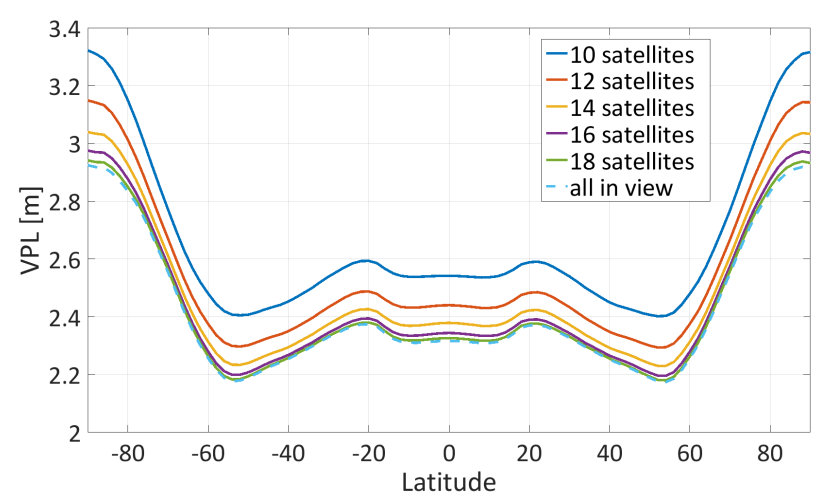

Figure 11: Vertical protection level averaged over all longitudes for different set sizes.

\section{SATELLITE SELECTION PERFORMANCE}

\section{Achieved Protection Levels}

In this section a short assessment of the proposed algorithm shall be given by comparing the results to optimal, bruteforce selected sets. A more intensive analysis on the overall performance of the new method as well as comparisons to established algorithms in terms of satellite selection will be provided in further studies. This paper is solely focusing on the comparison in terms of achieved GBAS protection levels in the vertical direction. Figure 9 therefore shows VPLs for roughly one day, comparing the obtained values using all satellites in view (green) with the best possible subset of 12 (red) as well as the heuristically selected 12 (blue). Singapore airport was chosen as simulated location in this case. The red graph is basically hidden due to the fact that the proposed heuristic selection method is achieving the exact same result more than $97 \%$ of the time. To get an idea of the actual error when using the heuristic Figure 10 shows only the difference to the brute-force selected best subsets. As can be seen there are only a few peeks where the heuristic is not able to find the actual best set, leading to $3.5 \mathrm{~cm}$ of difference in protection level at maximum. The average error over the whole simulated time is only minor in the range of $2 \mathrm{~mm}$.

\section{Computational Performance}

Regarding the computational expense Figure 12 gives an indication. The results are based on the same simulation which was shown before in the VPL comparison plots. On average the heuristic performs about 4-5 orders of magnitude faster than a simple brute-force check of all possible subsets, just because the number of constellations which need to be checked is drastically reduced as seen in the figure. The algorithmic overhead from sorting and selection operations on the other hand is minor compared to the matrix operations during VPL computation.

What can also already be seen is the fact that the computational load is hardly scaling with the complexity of the mere combinatorial problem of selecting $k$ out of $n$ without repetition. While the number of constellations which need to be checked using brute-force span over more than two 


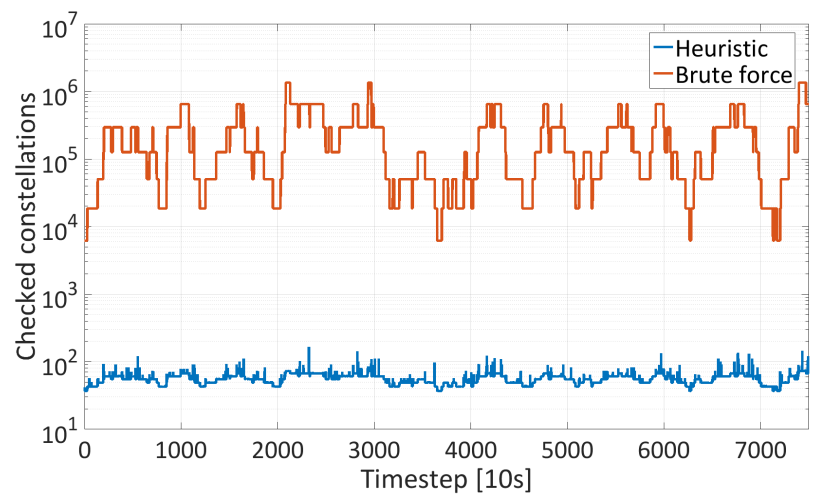

Figure 12: Comparison of computational effort for proposed heuristic and brute-force selected best set.

orders of magnitude in the example, the heuristic only needs 50 to 100 checks to find the result in most cases. This becomes especially important when considering the usage of even more than two constellations. Having for example 32 satellites to choose from instead of 22 increases the number of possible subsets by the factor of more than 1000 again, leading to $\left(\begin{array}{l}32 \\ 14\end{array}\right)=471,435,600$ combinations. Yet with vast computational power this becomes an issue for real time selection.

Finally, even if it was not part of this study, as computational performance was not the main focus of this paper, it shall be mentioned that there are ways to speed up the presented algorithm even more. All the performed manipulations on the S-matrix in this algorithm are only affecting a single satellite. This opens the possibility to utilize the matrix inversion lemma to make the satellite selection even more performant [12], as not the complete matrix has to be recalculated for every checked constellation. Furthermore some constellations are commonly checked more than once during the optimization process which enables a simple list of already checked sets to save some additional computation time.

\section{Consideration of Additional Constraints}

Another examined aspect was how well the subsets can fulfill additional secondary constraints.

The constraint of having a minimal number of two satellites per constellation in the selected set was fulfilled in all examined cases automatically. The number of Galileo satellites in subsets of e.g. size 14 was always between 3 and 9 (respectively 5 and 11 for GPS) with an average of $6.34 \pm 1.17$ satellites. This is almost exactly what one would expect taking into account the distribution of used GPS and Galileo satellites (31 and 27). At least two satellites are necessary as the time frames of different constellations are not aligned and therefore one satellite is already needed to estimate the time offset. Whether two satellites from another constellation are already reasonable, taken into account that only one would contribute to the position, could be subject of further studies.

Results from constraining the number of satellites to be

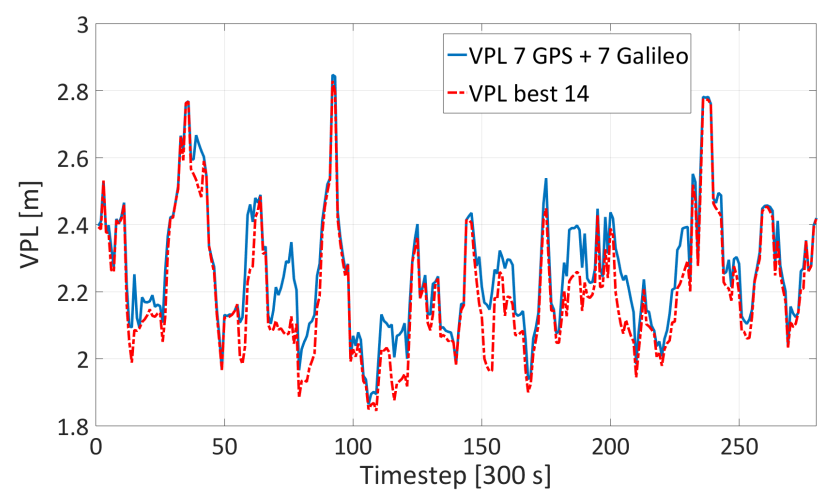

Figure 13: Comparison between unconstraint set of 14 and one of an equal number of GPS and Galileo satellites.

equal in a dual constellation GBAS are given in Figure 13. This can be interesting when considering aircraft only able to use one of the provided constellations. In this case the given corrections and integrity parameters should be optimized per system to prevent large fluctuations depending on the current overall constellation. The results show that the influence on the VPL obtained from the two separately optimized sets is highly fluctuating over time. While the average increase in VPL for the simulated day is only around $5 \mathrm{~cm}$, maxima reach up to $30 \mathrm{~cm}$. Nevertheless the common VPL variation over the day from changes in the constellation is about four times as large. Even this constraint should therefore be satisfiable without significantly reducing availability.

\section{CONCLUSIONS}

In this paper we presented a new method for fast, quasioptimal satellite selection. We showed that the algorithm bases on reasonable statistical assumptions and provides the necessary flexibility to be used in the GBAS context. Subsequent global simulations displayed that limiting the broadcast corrections to subsets of e.g. 14 satellites leads to only minor increases in terms of protection levels. The optimal selection of these subsets on the other hand becomes extremely costly in terms of computations with a growing number of available satellites. In GBAS the satellite selection has to be performed in real-time, thus an effective algorithm has to be used. The proposed method reduces the computational costs by orders of magnitude and achieves protection levels comparable to an exhaustive selection. Finally some additional constraints were considered and it was shown that these can be fulfilled without larger influence on the VPL. 


\section{References}

[1] Susumu Saito, Takayuki Yoshihara, Atsushi Kezuka, et al. "GAST-D Flight Experiment Results with Disturbed and Quiet Ionospheric Conditions". In: Proceedings of ION GNSS+. Tampa, US, 2015.

[2] M. Stanisak, A. Lipp, and T. Feuerle. "Possible VDB Formatting for Multi-Constellation / MultiFrequency GBAS". In: Proc. of the ION GNSS+. Tampa, FL, USA, 2015.

[3] ICAO NSP. GBAS CAT II/III Development Baseline SARPs. Tech. rep. International Civil Aviation Organization, 2010.

[4] RTCA DO-253C. Minimum Operational Performance Standards for GPS Local Area Augmentation System Airborne Equipment. Tech. rep. 253C. Radio Technical Commission for Aeronautics, 2008.

[5] M.-S. Circiu, M. Felux, S. Thölert, et al. "Evaluation of GPS L5 and Galileo E1 and E5a Performance for Future Multi Frequency and Multi Constellation GBAS". In: Jan. 2015 .

[6] Thomas Dautermann, Michael Felux, and Anja Grosch. "Approach service type D evaluation of the DLR GBAS testbed". English. In: GPS Solutions 16.3 (2012), pp. 375-387. ISSN: 1080-5370. DOI: $10.1007 /$ s10291-011-0239-3. URL: http: //dx.doi.org/10.1007/s10291-011$0239-3$.

[7] Miaoyan Zhang and Jun Zhang. "A Fast Satellite Selection Algorithm: Beyond Four Satellites". In: IEEE Journal of Selected Topics in Signal Processing 3.5 (Oct. 2009), p. 8. ISSN: 1932-4553. DOI: $10.1109 /$ jstsp . 2009 . 2028381. URL: http: / / dx . doi.org/10.1109/JSTSP. 2009.2028381.

[8] Dan Song, Pengfei Zhang, Guochao Fan, et al. "An Algorithm of Selecting more than Four Satellites from GNSS". In: International Conference on Advanced Computer Science and Electronics Information (ICACSEI 2013). 2013.

[9] Pratap Misra and Per Enge. Global Positioning System, Signals, Measurements, and Performance. 2nd Edition. Ganga-Jamuna Press, 2006.

[10] Nuria Blanco-Delgado and Fernando D. Nunes. "Satellite Selection Method for Multi-Constellation GNSS Using Convex Geometry". In: IEEE Transactions on Vehicular Technology 59.9 (Nov. 2010). ISSN: 1939-9359. DOI: 10.1109 / tvt . 2010. 2072939. URL: http:// dx. doi . org/ 10 . 1109/TVT.2010.2072939.

[11] Chan-Woo Park and Jonathan P. How. "METHOD AND APPARATUS FOR SELECTING OPTIMAL SATELITTES IN GLOBAL POSITIONING SYSTEM". Pat. US 6,727,850 B2. 2004.

[12] Makarand S. Phatak. "Recursive Method for Optimum GPS Satellite Selection”. In: IEEE, 2001.
[13] Duangduen Roongpiboonsopit and Hassan Karimi. "A Multi-Constellations Satellite Selection Algorithm for Integrated Global Navigation Satellite Systems". In: GITS 13.3 (July 2009). ISSN: $1547-$ 2450. DOI: 10 . $1080 / 15472450903084238$. URL: http : / / dx . doi . org / 10 . 1080 / 15472450903084238 .

[14] J Li, A Ndili, L Ward, et al. "GPS receiver satellite/antenna selection algorithm for the stanford gravity probe B relativity mission". In: Institute of Navigation (ION) National Technical MeetingVision 2010: Present and Future. San Diego, CA, 1999.

[15] P. D'Angelo, A. Fernandez, J. Diez, et al. "Performance and visibility analysis for different Galileo/GPS receivers with the GRANADA". In: Environment and Navigation Simulator. ENC/GNSS 2005 Conference, Munich, Germany (2005).

[16] Masahiko Kihara and Tsuyoshi Okada. "A Satellite Selection Method and Accuracy for the Global Positioning System". In: NAVIGATION, Journal of the Institute of Navigation, Volume 31, Number 1. 1984.

[17] Eurocae. MINIMUM OPERATIONAL PERFORMANCE SPECIFICATION FOR GLOBAL NAVIGATION SATELLITE GROUND BASED AUGMENTATION SYSTEM GROUND EQUIPMENT TO SUPPORT CATEGORY I OPERATIONS. Mar. 2013. 